\title{
Intelligent Hybrid Fault Diagnostic System
}

\author{
A.H. Mohamed \\ Solid State and Electronic Accelerators Dept \\ Atomic Energy Authority
}

\author{
M.H. El-Fouly \\ Solid State and Electronic Accelerators Dept \\ Atomic Energy Authority
}

\begin{abstract}
Recently, intelligent hybrid fault diagnostic systems are widely used to deal with the modern complex systems. It is found that, case based reasoning approach has proved its powerful as modern diagnostic technique. It can simplify the computation analysis required for diagnosis the complex systems. But, they still suffer from some limitations due to: (1) its inability to achieve a diagnosis for new faults that having no similar cases in its case library. (2) problems of storing the cases in the library and its effect on the complexity of the retrieving and adaptation processes. The proposed research introduces a new intelligent hybrid system that integrates the case based reasoning, neural network, and the genetic algorithm approaches to improve the performance of the CBR diagnostic systems. It incorporates the neural network into the case based system to diagnose the new faults. While, it uses the genetic algorithm to optimize the topology of the neural network and to select the optimum cases to be stored in the case library to manage its size. Therefore, the proposed hybrid diagnostic system can increase the accuracy, decrease the time and simplify the complexity of the retrieving and adaptation processes of CBR systems. The suggested system has applied for diagnosis the faults of a Wireless Network. This network communicates between a robot arm used for cleaning the solar cell panels of the complex PV systems, and its control centre as a case of study. The obtained results have proved good performance of the proposed hybrid diagnostic system in the practical sites.
\end{abstract}

\section{Keywords}

Case-based reasoning, genetic algorithm, neural network, fault diagnosis, and PV System.

\section{INTRODUCTION}

In recent years, there has been great importance for the fault diagnostic case based reasoning (CBR) systems. They can reuse and adapt the past solved cases and experiences in their case library to find the needed solution for similar new problems. On the other hand, they have some drawbacks such as:

1. Their inability to find diagnosis for the new faults that are not having similar stored cases in the case library $[1,2]$.

2. Increasing the number of the stored cases in the case library causes storage size problems. This increases the size of the case library, delay and complexity of the retrieving and adaptation processes [3-5].

To overcome these limitations, the proposed system introduces the using of $\mathrm{NN}$ to diagnose the new faults.
Because, it is found that, the neural networks (NNs) have proved their great success for diagnosis the new faults. Besides, the suggested system can use the genetic algorithm to optimize the topology of the Proposed NN. Also, GA optimizes the best cases to be stored in the case library for maintaining the CBR systems. Many successful systems have been developed to solve optimization problems for the engineering systems using the genetic algorithms.

From the review, it is found that, many hybrid systems integrating a CBR system with a neural network have been developed to improve the CBR's reasoning process [6,7]. For examples, a hybrid system proposed by Lee, et al. uses a neural network to improve the CBR adaptation process [8]. While, other researchers have concerned eliciting symbolic knowledge representation from a trained neural network [9$11]$.

On the other side, some hybrid systems of GA and CBR introduce great potential for many applications. For enhancement of matching and ranking procedure of CBR, GA is used to find an optimal weight vector of the attributes in case indexing and retrieving processes [12-15].

While, the present research introduces an intelligent hybrid system that integrates the CBR with both $\mathrm{NN}$, and GA to improve the performance of its diagnostic systems in novel view.

The reminder of this paper is organized as: Section 2 represents the CBR systems. Section 3 describes an overview of the neural networks. Section 4 deals with the genetic algorithm. Section 5 presents the proposed systems. Section 6 describes the applicability of the proposed system for diagnosis a robot arm used for cleaning the solar cell panels of the complex PV systems as a case of study and its evaluation. While, section 7 represents the conclusion.

\section{CASE BASED REASONING}

$\mathrm{CBR}$ is one of the most promising techniques to handle many attributes that are complex and unstructured information of the problem [16]. It is a problem solving technique that drives the solution based on remembering the past cases and using the similar cases for the new problem. The reasoning in the CBR systems is described as a cyclical process comprising of the four - Re's. These are: (1) retrieve the most similar case(s), (2) re-use the case to solve the problem, (3) revise the proposed solution if necessary and (4) retain the case and its solution to the case base as shown in fig. (1) [17].

Many CBR systems have great success in many application areas such as diagnosis, design, planning [18-20]. 


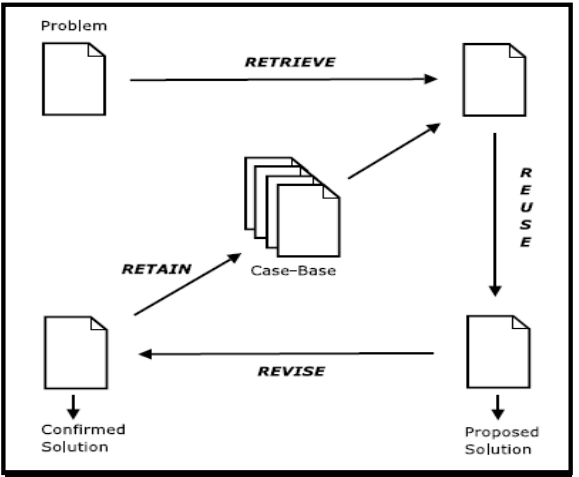

Fig. (1): A simple structure of CBR

\section{NEURAL NETWORKS}

Neural networks (NNs) are composed of many connected simple neurons as shown in fig. (2). (NNs) are representational and computational models processing information in a parallel distributed manner [21]. They are very useful for solving complex problems where the relationships between input and output data are not very well known, such as pattern and speech recognition, machine vision, robotics, signal processing, classifications and diagnosis [22]. Feed-forward and recurrent are two major classes of artificial neural networks. It is found that, feedforward neural networks are the most common type usually used as representational models trained using learning rules based on a set of input-output sample data. Back-propagation (BP) algorithm is a popular learning method widely used [23, 24].

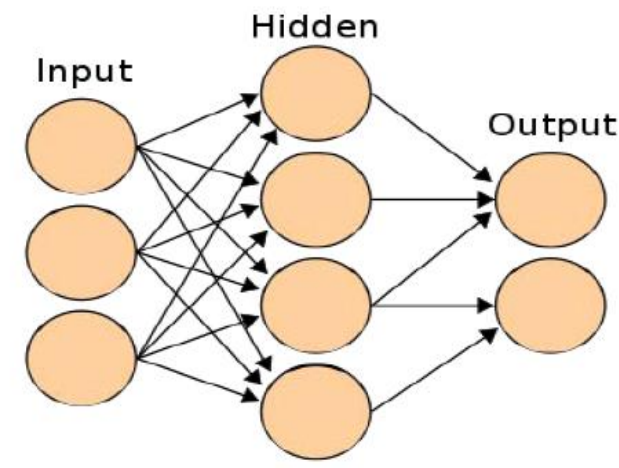

Fig.(2): The Structure of a Neural Network

\section{GENETIC ALGORITHMS}

Genetic Algorithms (GAs) are robust search and optimization techniques that were developed based on ideas and techniques from genetic and evolutionary theory. Beginning with a population of chromosomes, a genetic algorithm chooses parents from which generates the offsprings using crossover and mutation operators. The chromosomes are evaluated using a fitness function to determine their "fitness." According to the principle of survival of the fittest, the more adaptive chromosomes are kept to construct a new population [25]. The new population replaces the old one, and the whole process is repeated until the termination conditions are satisfied. A typical genetic process is described in fig. (3) [26].

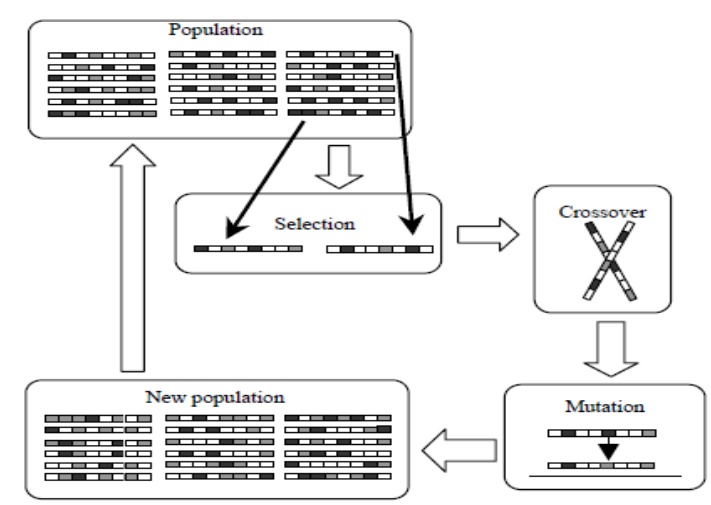

Fig. (3): A typical genetic process

\section{PROPOSED INTELLIGENT HYBRID DIAGNOSTIC SYSTEM}

Modern technology provides different complex machines and equipments used all over the world. Fault diagnosis of these modern systems is very complex, and sometimes become unmanaged or impossible task using the traditional diagnostic methods. In the recent years, case based systems have proved their success in solving different fault diagnostic problems. But, they are able to diagnose the faults have similar cases for those stored in the case library only. They suffer from great complexity and high costly time and money when applied for the modern complex systems.

The proposed system introduces new intelligent hybrid system to overcome the previous limitations. It can diagnose all the faults face the diagnostic system. CBR module can deal with the similar stored cases and incorporates the neural network (NN) can diagnose the new faults. Besides, the proposed intelligent system can use two genetic algorithms to solve two main problems. (1) optimizing the topology of the proposed NN. (2) optimizing the cases to be stored in the library and the others to be deleted.

The performance of the neural network depends mainly on its internal topology. But, achieving its topology factors is still determined by the trial and error approach and the subjectivity of designer. This may not guarantee the optimum topology.

So, optimizing this topology is very important task. The first GA operates at off-line to optimize the NN's topology. It can select the most important feature subsets, and determine the optimal number of hidden layers and neurons for the neural networks.

The encoded chromosomes of the first GA represented by the number of hidden layers, numbers of neurons, and ordering. The population has 30 chromosomes is initialized into random values. While, its fitness function is the average deviation between the expected and the determined values of costs. This GA operates the single crossover and the flip mutation iterates determine the optimum topology or run 50 times till the stopping conditions are satisfied. The results of this GA provide an optimized topology of the proposed neural network that in our present case has three layers with 28 neurons.

Figure (4) describes the procedure of the operation of the proposed system at run-time, as:

1. Getting the new case properties. 
2. Start searching the case library using the k-nearest neighbor retrieval algorithm to find most similar case(s) for the problem at hand.

a. If it is (they are) found, the system retrieves these similar cases and rank them. Then, adapt the highest rank case using the transformation adaptation technique.

b. Otherwise, if the proposed system fails to find similar stored cases, it uses $\mathrm{NN}$ to have the diagnosis. It uses the Back propagation learning (BPL) algorithm for the training process. BPL works by making modifications in weight values starting at the output layer then moving backward through the hidden layer of the network.

3. Either the fault has been diagnosed by CBR or by NN, it is represented as a new case that is required to be stored in the case library. In this moment, the proposed system uses the second genetic algorithm (GA) to evaluate this new case compared to the stored similar cases. So, it can optimize the best cases needed to be still stored or the others to be deleted.

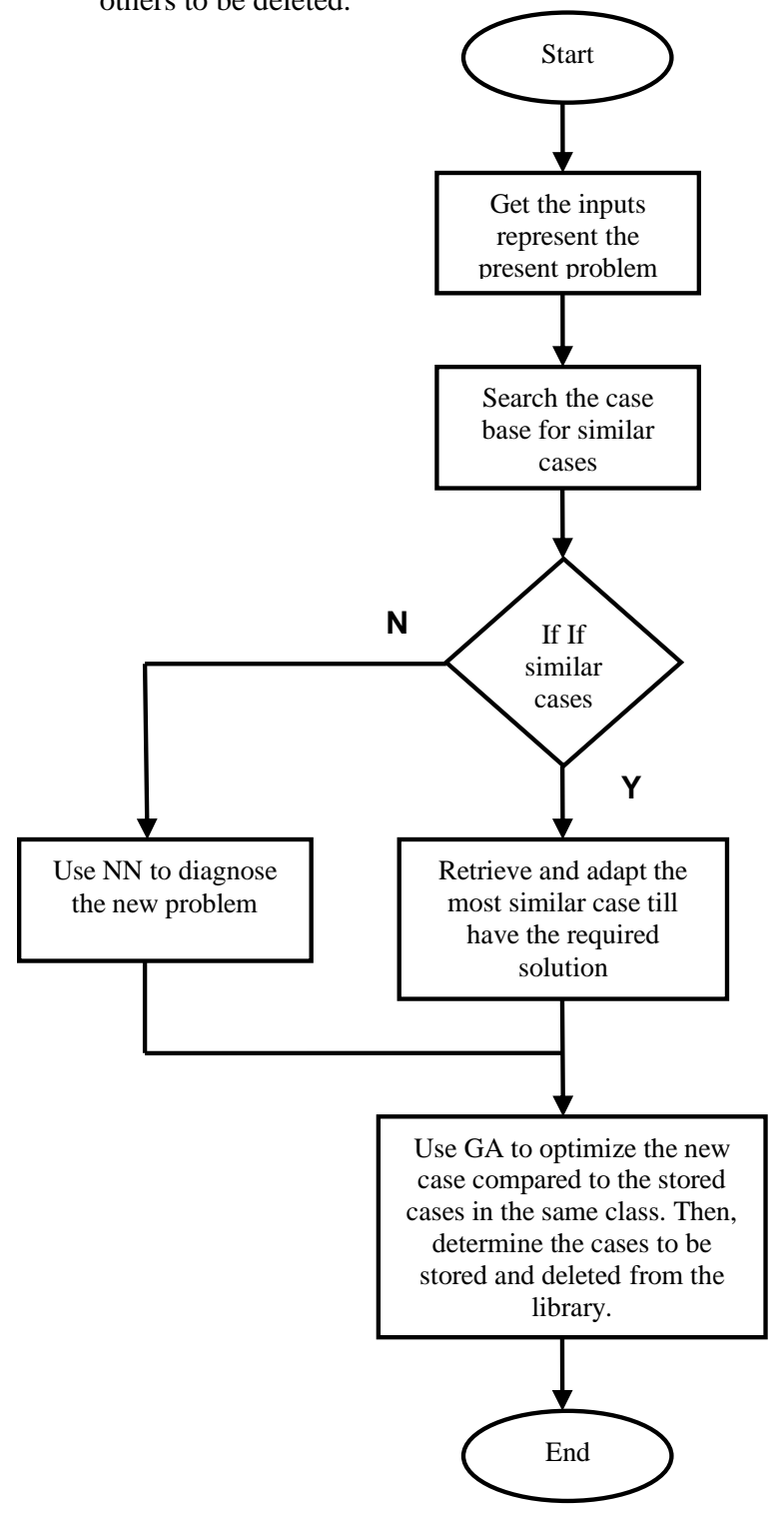

Fig.(4): Main idea of the proposed system
Genetic Algorithm computes all the coverage values of the cases in the library, and selects the case with the highest coverage as the representative similar cases for that class.

The genetic algorithm used in the proposed system can be implemented as:

1. The new case and its similar stored cases in the library represent the chromosomes for the population of the GA. They encoded as real values. Its population size $=30$. If the case library have more than 30 cases similar for the new case, the suggested system selects randomly 29 cases from the class of problem' cases and completing the first population with the new solved case.

2. Selection Methodology: The proposed system uses the roulette-wheel selection scheme. It is a fitnessproportionate selection form used to select the suitable offspring for the next generation.

3. Crossover: Proposed GA uses a single point crossover. The proposed crossover probability is: 0.6.

4. Mutation: Inversion mutation is applied to the offspring. The mutation rate is: 0.02 .

5. The fitness function of the system is represented as:

$$
F(x)=\sum_{i=1}^{n}\left(w_{i} x_{i}\right)
$$

Where $\mathrm{x}=$ instant of a feature in the case, $\mathrm{n}=$ total no. of features in the cases, w=weight of the importance of the feature in the problem.

6. The GA terminated either by achieving the solution or by run 50 iterations.

However, the proposed system can optimize the size of the cases in the case library. Therefore, the performance of the indexing, retrieval and adaptation processes can be improved.

\section{APPLICABILITY OF THE PROPOSED SYSTEM AND ITS RESULTS}

The proposed system can be applied for diagnosis different types of faults' devices or systems. In the present research, it has been applied for diagnosis the faults of a simulated Wireless Network that can be used in communicating between the control centre and a robot arm. This arm is used for cleaning the solar cell panels of the complex PV systems.

The proposed system divides this wireless network system into four parts: power, transmitter, control, and receiver units. Suggested system has used a case base contains 554 cases, 389 for training and 165 cases for testing. The power unit has 93 cases. The transmitter has 122 cases. The control unit has 208 cases. The receiver unit has 131 cases.

Its obtained results, a traditional case based and a traditional neural network fault diagnostic systems' results are compared $[18,27]$. Table (1) represents this comparison. From the achieved results, it is found that, the proposed system proves its goodness by increasing the accuracy of the fault diagnostic process and decreasing the run-time of its operation. As, the proposed system increases the accuracy of the diagnostic system to $98.3 \%$ and its run time $=2.6 \mathrm{~min}$. compared to the traditional case based diagnostic system that has average accuracy $=89.1 \%$ while its run time $=5.0 \mathrm{~min}$. Also, it can improve the diagnostic process relative to the traditional 
neural network diagnostic system that has average accuracy $=$ $80.2 \%$ while its run time $=6.1 \mathrm{~min}$.

On the other side, evaluating the storage size of the case library and the performance of the proposed system with the traditional case based fault diagnostic system without using the genetic algorithms are shown in Table (2). It is found that, after 50 run-sessions for the compared systems, the case library increased 83 cases from 554 to 637 when using the diagnostic system without the GA. While, it increased 15 cases only by increasing the cases in the library to 569 cases when integrates the GA into the NN- CBR diagnostic system. This derives to decrease the complexity and run-time of the retrieving and adaption processes. Besides, it has decreased the run-time and accuracy comparing the results obtained from the proposed system with those getting without using the GA. From the previous results, it is found that, the proposed system has proved its goodness and significant performance when applied for real-time application.

Table (1): A comparison between the proposed diagnostic system, a traditional case based, and a traditional neural network system

\begin{tabular}{|c|c|c|}
\hline Diagnostic System & $\begin{array}{c}\text { Average Accuracy } \\
\text { of the unit }(\%)\end{array}$ & $\begin{array}{c}\text { Average Time } \\
\text { (min.) }\end{array}$ \\
\hline \multicolumn{3}{|c|}{ Proposed System } \\
\hline Power unit & 98.2 & 1.3 \\
\hline Transmitter Unit & 96.4 & 1.7 \\
\hline Control Unit & 95.5 & 2.2 \\
\hline Receiver Unit & 96.1 & 2.6 \\
\hline \multicolumn{3}{|c|}{ Traditional CBR Fault Diagnostic System } \\
\hline Power unit & 87.2 & 4.7 \\
\hline Transmitter Unit & 86.4 & 4.0 \\
\hline Control Unit & 85.7 & 3.7 \\
\hline Receiver Unit & 85.5 & 3.6 \\
\hline \multicolumn{3}{|c|}{ Traditional Neural Network Diagnostic System } \\
\hline Power unit & 80.2 & 5.4 \\
\hline Transmitter Unit & 80.4 & 5.8 \\
\hline Control Unit & 77.3 & 5.5 \\
\hline Receiver Unit & 79.5 & 5.8 \\
\hline
\end{tabular}

Table (2): A comparison between the proposed and traditional case based diagnostic systems

\begin{tabular}{|l|c|c|c|}
\hline $\begin{array}{l}\text { Diagnostic } \\
\text { System }\end{array}$ & $\begin{array}{c}\text { Case Library } \\
\text { Size }\end{array}$ & $\begin{array}{c}\text { Average } \\
\text { Accuracy of } \\
\text { the system } \\
(\%)\end{array}$ & $\begin{array}{c}\text { Average } \\
\text { Time (min.) }\end{array}$ \\
\hline $\begin{array}{l}\text { Proposed } \\
\text { System } \\
\text { without the } \\
\text { genetic } \\
\text { algorithm }\end{array}$ & 637 & 97.1 & 3.6 \\
\hline $\begin{array}{l}\text { Proposed } \\
\text { System } \\
\text { with the } \\
\text { genetic } \\
\text { algorithm }\end{array}$ & 569 & 98.5 & 2.8 \\
\hline
\end{tabular}

\section{CONCLUSION}

Although case based reasoning systems have proved its goodness for the diagnosis processes in many fields, they suffer from a failure when it faces with new faults that is not stored in their case library. On the other hand, neural networks have a proven their ability in the area of fault diagnosis. After being trained, they contain expert knowledge and can correctly identify the different causes of faults.

Therefore, the proposed system introduces a new intelligent hybrid case based fault diagnostic system. It can incorporate the neural network diagnosis algorithm into CBR system to deal with the unmatched new cases with the stored cases in the case library. Besides, it uses genetic algorithm to optimize the cases to be added or deleted in the case library to manage the size of the case library.

However, the proposed system can manage the problems of storage the cases in the library. The uncontrolled growth of the stored cases causes many problems appeared in the memorization and the complexity of the retrieval and adaptation processes.

The proposed system is applied for diagnosis of a Wireless Network that can be used in the operation of a robot arm for cleaning the solar cell panels of the complex PV systems. The obtained results indicate that incorporating a neural network and the genetic algorithm into the case based system for diagnosis the wireless network can increase its accuracy and decrease the diagnostic time, the size of its library and complexity of the retrieving and adapting processes.

\section{REFERENCES}

[1] Teodorovic, D., Šelmic, M., and Teodorovic, L. M. 2013. "Combining Case-Based Reasoning with Bee Colony Optimization for Dose Planning in Well Differentiated Thyroid Cancer Treatment", Expert Systems with Applications, 40: 2147-2155.

[2] Beddoe, G., Petrovic, S. , and Li, J. 2009. "A Hybrid Meta-Heuristic Case-Based Reasoning System for Nurse Roistering", Journal of Scheduling 12 (2): 99-119.

[3] Luger, F. G. and Stubblefield, A. 2011. Addison Wesley Longman Inc., Third Editions, USA, pp.125-140.

[4] Oman, S. and Cunningham, P. 2001. "Using Case Retrieval to Seed Genetic Algorithms", International Journal of Computational Intelligence and Applications, 1(1): 71-82.

[5] Burke, E. , MacCarthy, B., Petrovic, S. and Qu, R. 2006. "Multiple-Retrieval Case-Based Reasoning for Course Timetabling Problems", Journal of Operations Research Society, 57 (2):148-162.

[6] Aamodt A. and Plaza, E. 1994. "Case-Based Reasoning: Foundational Issues, Methodological Variations, and System Approaches", Artificial Intelligence Communications, 7:39-52.

[7] Madureira, A., Santos, J. and Pereira, I. 2009. A Hybrid Intelligent System for Distributed Dynamic Scheduling, Natural Intelligence for Scheduling, Planning and Packing Problems, Studies in Computational Intelligence, eds. R.Chiong, and S.Dhakal, 250: 295-324.

[8] Lee, C.-H. L. , Liu, A. and Huang, H.-H. 2010. "Using Planning and Case-Based Reasoning for Service 
Composition", Journal of Advanced Computational Intelligence and Intelligent Informatics, 14 (5):450-456.

[9] Kwang H. I. and Sang, C. P. 2007. "Case-Based Reasoning and Neural Network Based Expert System for Personalization", Expert Systems with Applications 32:77-85.

[10] Chen D. and Burrell, P. 2001. "Case-Based Reasoning System and Artificial Neural Networks: A Review", Neural Computation and Application, Springer-Verlag London Limited, 10:264-273.

[11] Musa, A. G., Daramola, O., Owoloko, A., Olugbara, O. 2013. "A Neural-CBR System for Real Property Valuation", Journal of Emerging Trends in Computing and Information Sciences, 4(8):611-622.

[12] Mala, D. J., Elizabeth S. R. and Mohan, V. 2008. "Intelligent Test Case Optimizer - An Automated Hybrid Genetic Algorithm based Test Case Optimization Framework", International Journal of Computer Science and Applications, 1(1):51-55.

[13] Sànchez-Marrè1, M., Gibert, K. , Vinayagam, R. K. , S.Villanueva, B. 2014. "Evolutionary Computation and Case-Based Reasoning Interoperation in IEDSS through GESCONDA, International Environmental Modeling and Software Society (iEMSs), 7th Intl. Congress on Env. Modeling and Software, San Diego, CA, USA, Daniel P. Ames, Nigel W.T. Quinn and Andrea E. Rizzoli (Eds.).

[14] Dou, J. , Chang, K.-T., Chen, S., Yunus, A. P., Liu, J.-K., Xia H. and Zhu, Z. 2015. "Automatic Case- Based Reasoning Approach for Landslide Detection: Integration of Object-Oriented Image Analysis and a Genetic Algorithm, Remote Sensing, 7:4318-4342.

[15] Ahn, H., Kim, K. , and Han, I. 2006. "Hybrid Genetic Algorithms and Case-Based Reasoning Systems for Customer Classification", Expert System, 23:127-144.

[16] Minor, M., Bergmann, R., and Görg, S. 2014. "Casebased Adaptation of Workflows", Information Systems, 40: $142-152$.

[17] Cordier, A., Mascret, B. and Mille, A. 2013. "Dynamic Case Based Reasoning for Contextual Reuse of Experience", Case-Based Reasoning Workshop, Cindy Marling ed. Alessandria, Italy, 69-78.
[18] Prentzas, J. and Harzilygeroudis, I. 2009. "Combination of Case-Based Reasoning with other Intelligent Methods", International Journal of Hybrid Intelligent systems, 6(2809): 189-209.

[19] Plaza, E. and Mcginty, L. 2013. "Distributed Case-Based Reasoning, The Knowledge Engineering" Review, Cambridge University Press,72(4):30-37.

[20] Hidayah, I. , Syahrina, A. and Permanasari, A. E. 2012. "Student Modeling using Case-Based Reasoning in Conventional Learning System", (IJCSIS) International Journal of Computer Science and Information Security, 10(10):21-27.

[21] Azuaje, F., Dubitzky, W., Lopes, P., Black, N. and Adamsom, K. 2007. "A Neural Network Approach", Artificial Intelligence in Medicine, 15: 275-297.

[22] Gong, L. , Liu, C., Li, Y. and Yuan, F. 2012. "Training Feed-forward Neural Networks Using the Gradient Descent Method with the Optimal Stepsize", Journal of Computational Information Systems 8(4):1359-1371.

[23] HUK, M. 2012. "Back-Propagation Generalized Delta Rule For The Selective Attention Sigma-If Artificial Neural Network", International Journal of Applied Mathematics and Computer Science, 22 (2):449-459.

[24] Zhao, Q. and Ye, F. 2013. "A New Back-Propagation Neural Network Algorithm for a Big Data Environment Based on Punishing Characterized Active Learning Strategy", International Journal of Knowledge and Systems Science, 4(4): 32-45.

[25] Brill, F.Z., Brown, D.E., Martin, W.N. 2012. "Fast Genetic Selection of Features for Neural Network Classifiers", IEEE Transactions on Neural Networks, 3:324-328.

[26] Yang, J. and Honavar, V. 2012. "Feature Subset Selection Using a Genetic Algorithm”, IEEE Intelligent Systems, 13:44-49.

[27] Gharehchopogh, F. S., Molany M. and Mokri, F. D. 2013. "Using Artificial Neural Network in Diagnosis of Thyroid Disease: A Case Study", International Journal on Computational Sciences and Applications (IJCSA), 3(4):49-61. 